\title{
On some classes of retro Banach frames in Banach spaces
}

\author{
MAYUR PURI GOSWAMI
}

\author{
Date of Receiving : $\quad 13.02 .2018$ \\ Date of Revision : $\quad 27.07 .2018$ \\ Date of Acceptance : 02.08 .2018
}

\begin{abstract}
In this paper, we study certain classes of retro Banach frames, namely, retro bi-Banach frame, retro Bessel sequences. In the sequel, we characterize retro Bessel sequences with the help of examples and establish some relations between retro Bessel sequence and retro Banach frame. Finally, results on the existence of retro bi-Banach frames have been obtained.
\end{abstract}

\section{Introduction}

In 1952, frames for Hilbert spaces were introduced by Duffin and Schaeffer [5], in the context of non-harmonic Fourier series. Nowadays frames play a crucial role in various branches of pure and applied mathematics and engineering sciences. In 1988, Hilbert frames were generalized to Banach spaces by Feichtinger and Gröchenig [6], called atomic decomposition. For more on Banach frame literature one may refer to $[2,11,3]$.

In 2004, Jain et al. [10] extended Banach frames to the conjugate Banach spaces and introduced retro Banach frames. Recently, Shalu Sharma [15], introduced a notion of the bi-Banach frame as a pair of Banach frame and retro Banach frame. Motivated by the paper [15], Goswami et al. [7] defined the retro bi-Banach frame in conjugate Banach spaces and established some relations of retro bi-Banach frame with some existing notions in Banach frame theory. Bessel sequences, another important notion in the frame theory were studied in $[12,13]$. In this paper, we defined retro Bessel sequences and obtained some results regarding construction of retro Bessel sequences and retro bi-Banach frames. Our results extend some previous results proved for $G$-Banach Bessel sequences [13] and strong retro Banach frames [9].

2010 Mathematics Subject Classification. 42C15, 46B15.

Key words and phrases. Banach frame, Retro Banach frame, Retro Bessel sequences.

The author indebted to the anonymous referees for their helpful comments and suggestions toward the improvement of the paper.

Communicated by: Geetika Verma 